\title{
XLIV. Notes on the "break" of a magneto or induction-coil
}

\section{Norman Campbell Sc.D.}

To cite this article: Norman Campbell Sc.D. (1919) XLIV. Notes on the "break" of a magneto or induction-coil , Philosophical Magazine Series 6, 37:221, 481-494, DOI: 10.1080/14786440508635908

To link to this article: http://dx.doi.org/10.1080/14786440508635908

曲 Published online: 08 Apr 2009.

Submit your article to this journal $\sqsubset \pi$

Џ Article views: 2

Q View related articles $\asymp$ 
XLIV. Notes on the "Breali" of a Magneto or InductionCoil. By Norman rampbeli, Sc.D.*

NoTE - The work described in these notes was carried out at the National Physical Laboratory under the direction of the Advisory Committee for Aeronautics. The results have been communicated in a confidential report to the Internal Combustion Engine Sub-Committee of that Committee, who have now given their consent to the publication of any portions which appear of pure scientific interest.

\section{(1) Introduction.}

T $T$ is well known that if the greatest possible efficiency is 1 to be obtained from a magneto or induction-coil, that is, the greatest possible ratio of the maximum secondary potential to the primary current broken, it is necessary to avoid sparking between the terminals at which the primary current is broken. No elaborate theory is necessary to explain the loss of elficiency due to sparking. In the first place the spark involves the dissipation of some of the electromagnetic energy originally present in the primary current which would otherwise lave been available for conversion into electrostatic energy of the secondary. In the second place the passage of the spark, even if it involved no loss of energy, would prolong the time which elapses between the first decrease of the primary current, as the contact opens, and its total cessation; very general considerations will show that, if this time is so prolonged as to become an appreciable fraction of the period of the oscillations excited, a loss of efficiency will usually follow.

It is always possible to suppress the spark at the primary break by inserting a condenser of sufficient capacity in parallel with the separating terminals. Until an adequate theory of the induction-coil was developed, chiefly by the work of Prof. Taylor Jones and his collaborators $t$, it seems to have been believed that the suppression of the spark was the only useful function of the primary condenser; and that if the suppression could be achieved by any other means, such as an increase in the speed of separation of the terminals, the addition of capacity to the primary circuit would be unnecessary. This view was, of course, largely based on the classical experiments of Lord Rayleigh $f$, who showed

* Communicated by the Author.

$\dagger$ Phil. Mag. Jan. 1909, p. 28: Nov. 1911, p. 706 : April 1914, p. 580; Jan. 1915, p. 1; Aug. 1915, p. 224; A pril 1917, p. 322.

$\therefore$ Phil. Mag. ii. p. 581 (1901).

Phil. Mag. S. 6. Vol. 37. No. 221. May 1919. 2 L 
that, in the absence of a primary condenser, the maximum secondary potential could be very considerably increased by increasing sufficiently the speed of the break. This conclusion was confirmed by Prof. Taylor Jones; but the further conclusion that, if the speed of the break were increased sufficiently, as great a secondary potential could be obtained without a condenser as with one, was not confirmed. Theory and experiment alike show that, oven if the break is perfect and there is no sparking, there is an optimum value for the primary capacity, depending on the other constants of the inductive circuits; and that the ase of a capacity either greater or less than this optimum value involves some sacrifice of efficiency.

Accordingly, in order that the greatest possible efficiency should be obtained, it is necessary that this optimum primary capacity should not be less than that which is necessary to prevent sparking ; for otherwise, if a capacity is used large enough to prevent sparking, there will be a loss of efficiency, small but important, due to the use of a primary capacity greater than the optimum. In all magnetos which have been examined, and probably in all inductioncoils with all forms of break, this necessary condition is not fulfilled; the optimum capacity is less than that required to prevent sparking. Mo:t magneto manufacturers are aware that the secondary potential developed increases steadily as the primary capacity is diminished up to the point where: sparking begins; the capacity of the primary condenser which it is necessary to use is determined wholly by the necessity for avoiding sparking.

It becomes of importance, therefore, to inquire whether the capacity necessary to avoid sparking might not be diministed by changing some other characteristic of the break. The only characteristic which seems to have been considered in this connexion is the speed of separation of the terminals. According to the view which seems generally accepted, the spark between the separating terminals occurs because the rate at which the potential difference between those terminals increases as a result of the interruption of the current is greater than the rate at which the spark potential of the gap between the terminals increases with the distance between them*. The primary condenser stops

* This theory is formally stated and supported by some experiments in a paper by U. Wiesinger, Ann. d. Phys. Iv. 6, p. 401 (1918); but it must have occurred to everyone who has paid any attention to the matter. It will be seen later that the conditions of the experiments by which Wiesinger supported his theory weie not those obtaining in the primary circuit of a magneto or induction-coil. 
sparking because it decreases the rate of rise of the potential difference between the terminals. It is to be expected that sparking would also be stopped by an increase in the rate of rise of the spark potential, which would accompany an increase in the rapidity with which the terminals separate.

However, Lord Rayleigh's experiments seemed to indicate that any practical application of the second alternative is out of the question, becuuse the necessary rate of separation is so great. He found that no advantage was gained by increasing the speed of separation up to that of a pistol bullet, and that it was only when a rifle bullet was employed to cut the primary circuit that any increase in the secondary potential was obtained. The critical velocity would doubtless depend on the constants of the circuits and on the primary current broken (for these determine the rate of rise of potential), but in the case investigated it was so much greater than anything which could be obtained with a mechanical break that research in this direction bas not seemed promising.

It may be noted that, if we knew the rate of rise of potential and the relation between the spark potential and the distance between the contacts, the determination of the critical velocity would permit an experimental test of the theory. The rate of rise of potential can be determined with any arrangement for measuring the wave-form, but the relation between the spark potential and the distance between the contacts is less easy to determine satisfactorily for the very short distances which are here involved. It is known that, as the distance is reduced, the spark potential diminishes to the "minimum spark potential" of about 350 volts at a distance somewhat less than $0 \cdot 1 \mathrm{~mm}$., that it subsequently increases and then falls again very rapidly when the distance becomes about $0.001 \mathrm{~mm}$. In the interval between $001 \mathrm{~mm}$. and $0 \cdot 1 \mathrm{~mm}$. the relation is so complicated that it is difficult to determine with the necessary accuracy.

(2) Experiments.--Some observations which have been made in the course of the work described in a recent paper* seem to throw some new light on the question; and although they are not sufficient to answer all the questions that can be raised, they seem to point to conclusions of sufficient practical importance to justify their record.

Observations that seem to indicate that the theory in its present form is not adequate to explain all the facts can be

* Phil. Mag. vol, xxxvii. ser. 6, p. 284, March 1919. 
made very readily on any magneto. If we remove the magnets and bring the primary terminals outside the machine, we can supply from a battery a primary current which is independent of the speed*. Witl any given primary condenser and any given speed, it will be found that, if the current is below a certain limit, no sparking occurs, but that if it is increased above this limit, sparking appears. The limiting value can be fixed within comparatively narrow limits; in one experiment, for instance, it was found to lie certainly between 2.2 and $2.5 \mathrm{amp}$. Suppose now that we supply a current double the limiting value; then we shall very nenrly (but not quite) double the rate of rise of potential across the terminals. Suppose now we double the speed of the magneto shaft and so double the speed of separation of the terminals; then at any moment during the rise of potential the relation between the spark potential and the potential difference between the terminals should be exactly the same as it was at some corresponding moment with the original values of the current and the speed. This conclusion involves only the assumption that the rate of rise of potential is proportional to the current broken; it does not involve any assumption concerning the relation between the spark potential and the distance. Accordingly the current which is now broken in the primary should again be the limiting current; in other words, by doubling the speed of break we should expect very nearly to double the limiting current. But as a matter of fact we find that the limiting current is not donbled or nearly doubled by doubling the speed; no certain evidence could be produced that increasing the speed of the break 20-fold produces a change in the limiting current which is greater thin the error (10 or 20 per cent.) in the determination of that current. 'This conclusion appear's absolutely inconsistent with the theory which has been given of the circumstances which determine the occurrence of sparking.

(3) Effect of the electrodes. - The clue to this apparent failure of a theory, so obvious that it has not generally been

* The same experi:nent can be tried without disconnecting the primary circuit by making use of the fact that the primary current in a magneto is almost independent of the speed over a considerable range; it is usually possible within this range to change the speed 100 per cent. without changing the primary curvent more than 10 per cent.; and if we start on one slde of the maximum and decrense or increase the: speed through the maximum to the other side, we cun vary the speed more than 100 per cent. and regain the original value of the primary current. The value of the maximum current can be readily changed by changing the strength of the magnets. 
thought worth while to state it, is readily found if the intluence of the nature of the contacts at which the primary current is broken is examined. According to the theory we have been examining, the nature of the contacts should be almost without influence; for the spark potential is almost independent of the form and material of the terminals when the distance separating them is very small. But the crudest experiments will indicate that the nature of the contacts is a most important feature in determining the limiting current. In one matter indeed the influence is familiar to all who have used a magneto or induction-coil; we have only to roughen the surface of the contacts and sparking immediately occurs at the break which was sparkless before; the limiting current may be reduced "50 per cent. by scratching the surface and restored to its original value by polishing.

More systematic experiments indicated that, if the constants of the eircuits remained unchanged, the limiting current which could be broken without sparking always increased as the resistance of the contacts decreased. Some observations on the resistance of the contacts are described in a note at the end of this paper, but in general it may be said that the resistance of a contact can be decreased either by increasing the area of the surfaces in contact or the pressure between them. Thus in a certain circuit the contact fitted in a magneto would break without sparking a current of 4 amp.; a contact consisting of a ball $\frac{1}{4}$ in. in diameter pressed with a force of 50 grams weight against a steel plate would break only $2 \cdot 2 \mathrm{amp}$; if force was reduced to $1 \mathrm{gm}$. weight, the limiting current was barely $\frac{1}{2}$ amp. In no case could any relation be found between the limiting current and the speed of break, which in these observations could be varied from 300 to $62 \mathrm{~cm} . / \mathrm{sec}$. 'The influence of the material of which the contact was composed was not investigated.

(4) Formation of an arc.-The great variation of the limiting current with the resistance of the contact gives at once the clue to discrepancies which have been noted. The "spark" which is seen to occur at the broken contact is not a true spark, but an arc*. The discharge only occurs if the passage of the current between the contacts before the

* This conclusion might almost have been deduced from the mere appearance of the "spark." So far as we have observed, a luminosity confined to a narrow path between the contacts more or less straight, is never seen ; what appears when a primary circuit is broken is a "flash," such as occurs when steel strikes flint. It may be mentioned that several writers have called the spark at break an "arc" but they do not seem to have realized the implications of that term. 
break has caused such intense local heating that portions of the terminals hive been raised to the temperature at which notable thermionic emission begins, so that a discharge between them can be maintained by a potential difference quite inadequate to start a spark.

With the object of testing this explanation measurements were made on the rise of potential across the break as the terminals separated. The circuit broken was the primary of a magneto with a condenser of 1 mf. across the break; one terminal consisted of a $\frac{1}{4}$ in. steel ball attracted with a force of abont 50 grams weight to the end of the magnet which formed the other terminal. The limiting current was 1.2 amp. ; it was found that, so long as the current was less than this, the potential at any given moment after break was very nearly proportional to the current broken. Accordingly the rise of potential was first determined with a current less than the limiting vilue $(1.0 \mathrm{amp}$.$) ; if we$ multiply all the measured values of the potential by 1.5 we get a curve $A$ in fig. 1, which represents the rise of potential

Fig. 1.

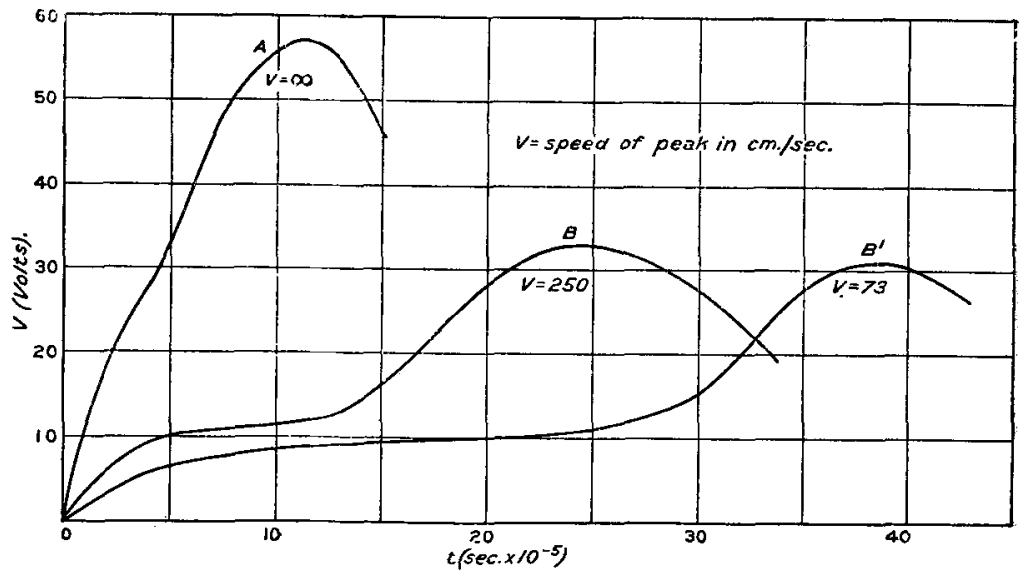

which would follow when a current of $1.5 \mathrm{amp}$. is broken, if there were no arc formed. Curves $B$ and $B^{\prime}$ give the measured rise of potential when a current of $1.5 \mathrm{amp}$. is broken, and the speed of the separation of the terminals is $250 \mathrm{~cm} . / \mathrm{sec}$. and $73 \mathrm{~cm} . / \mathrm{sec}$. in $B$ and $B^{\prime}$ respectively. It will be seen that from the very first instant the rate of rise is much less rapid in $B$ and $\vec{B}^{\prime}$ than in $A$; that a stage 
is soon reached in which the potential is nearly constant for a considerable time, longer with the slower separation; and that subsequently the potential rises rapidly and attains a maximum, nearly independent of the speed of separation, at a time after the beginning of the rise which is again nearly independent of the speed of separation and "lso nearly equal to the time that curve A takes to rise from zero to its maximum.

The form of the curves is exactly what we should expect if an are is formed, and utterly unlike that which is characteristic of the passage of a spark (see p. 492). The initial rate of rise of potential is small because the current is not broken when the contacts separate but is maintained by the arc between the hot contacts. The constant potential which persists for some time is very nearly that of the battery supplying the primary current (10 volts). At the point where the second rise of potential begins the arc ceases, and the rest of the curve represents approximately that which would have been obtained if a current, equal to that flowing in the arc, had been broken at the first separation of the contacts.

Many more experiments would be necessary to establish the complete accuracy of all the statements that have been made and to work out in detail the quantitative relations which are suggested. But the observations which have been described are sufficient to show that the discharge which occurs when the "flash" is seen at the broken contact is of the nature of an are rather than a spark, and that it is conditioned by the heating of the electrodes.

And it is now apparent why the "flash" may be prevented by decreasing the rate of rise of potential, but not by a corresponding increase in the rate of separation of the terminals. The potential of the battery (or, in the case of the magneto, that due to the rotation of the armature in the magnetic field) is not sufficient to maintain the arc permanently. If this potential is only 10 volts, then, however slowly the contact is opened, the current ceases a very small fraction of a second after the distance between the contacts has reached a value which is certainly less than $0.01 \mathrm{~mm}$. The energy dissipated in the discharge is not sufficient to maintain the electrodes at the high temperature necessary for the arc-discharge. On the other hand, the passage of the discharge and the dissipation of energy which accompanies it delay the cooling of the electrodes, and they delay it the longer the greater is the potential difference maintaining the discharge. Accordingly the more slowly 
the potential rises after the separation of the contacts, the shorter will be the time which elapses before the arc is extinguished by the cooling of the terminals; indeed, if it rises sufficiently slowly the terminals will have cooled before the potential has reached a value which is sufficient to give a "flash," even if the electrodes were hot. In that case the break will be sparkless. On the other hand, it is known that the potential necessary to maintain an arc does not increase rapidly with the distance between the electrodes; and we should expect, therefore, as we find, that the maintenance of an arc for an appreciable time, which is probably necessary for the appearance of the "flash," would depend very greatly on the rate of rise of potential and very little on the rate of separation of the terminals.

A further examination of the curves produces evidence in support of the conclusion that the final cessation of the arc depends on the time which elapses since the break as well as on the distance to which the electrodes have separated. For though the time the arc lasts is longer for the slower separation, the distance of the electroles when it ceases is greater for the quicker separation $(0.30 \mathrm{~mm}$. as against $0 \cdot 19)$. Since the potential when the arc ceases is almost the same in the two cases, this difference can only mean that the time as well as the distance is important. "The maintenance of the arc retards the cooling of the electrodes, but since the potential difference is not sufficient to keep the electrodes permanently hot, the instant when the arc ceases is determined in part by the losses due to radiation and conduction.

Again, we can obtain a minimum estimate of the speed of break which would be necessary to obtain a sparkless break simply by increasing the speed of separation. In order that this result should be attained, the separation must ve so rapid that the distance between the electrodes becomes so great that, even if the electrodes are still hot, a visible discharge cannot be produced with the potential available. Now curve B shows that the are can be maintained with a potential of 10 volts with the electrodes $0.30 \mathrm{~mm}$. apart, if they are sufficiently hot; accordingly mere speed will not prevent sparking unless the electrodes have separated $0.30 \mathrm{~mm}$. before curve $A$ has reached 10 volts (or less). The minimum estimate of the speed which will prevent sparking is, therefore, $30,000 \mathrm{~cm}$. $/ \mathrm{sec}$ - - a value very difficult to obtain in any mechanical break, though much less than the speed of Lord Rayleigh's pistol bullet. However, it must be remembered that the estimate is on all grounds merely a minimum estimate, and there is nothing to render 
it likely that the true limiting speed is not really much greater.

On the other hand, it should be noted that a sufficient increase in the speed of break may prevent arcing in another way ; the heating of the electrodes may not begin until the separation begins, and a reduction of the time of separation may reduce the time avialable for heating them to the requisite temperature. A reduction of arcing due to this cause is most likely to occur when, as in Lord Rayleigh's experiments, the circuit is broken by cutting a solid conductor; but it will also ocenr with a contact such as that employed in a magneto, where the terminals are held together by considerable pressure.

It is probably for this reason that it has been found that if, by the use of special cams, the contacts of a magneto are opened very slowly, flashing can be made to occur when it "ill not occur with the normal rate of opening. In the contacts used in these experiments, the pressure between the contacts berore opening was much less and the resistance of the contact much greater; heating began before the contacts separated. In such circumstances the limiting current appeared quite independent of the speed of separation within the range which could be observed (20 to $250 \mathrm{~cm} . / \mathrm{sec}$.).

Again, it is possible that the increase in the secondary potential which Lord Rayleigh found may be merely due to the fact that the time that the arc lasted was greatly reduced ; for if the interval between the first opening of the contacts and the total cessation of the current could be made very much less than the period of the osciliations excited, the loss of efficiency due to "sparking" would be greatly reduced. But this explanation is improbable. For, if it were correct, there would be a gradual increase in the efficiency as the speed of break was increased, whereas Lord Rayleigh found no increase whatever until the speed of a rifle bullet was reached. However, in the experiments described here there was some evidence that an increase in the speed of break, when there is sparling, produces some slight increase in the secondary potential developed; but when there is sparking the secondary potential varies so greatly at successive breaks that accurate measurements are difficult. In all the cases examined the secondary potential when there was sparking was at least 30 per cent. less than that obtained with a slightly smaller current which produced no sparking.

(5) The Perfect Break.-The conclusions to which these 
observations are directed is that the speed of the separation of the terminals is a relatively unimportant matter in the prevention of sparking. The speed doubtless exerts some influence, and a reduction of the speed much below that usually employed would probably be harmful; on the other hand, an increase, if sufficiently large, would doubtless be beneficial. But there is a wide range of speeds, inclading those usually employed, over which the speed has practically no effect. If the use of a smaller primary capacity is to be made possible, the improvement must be sought by reducing. the heating at the contart and pieventing the development locally of high temperatures which permit the formation of an are.

The exact conditions which tend to prevent arcing have not been studied in detail; it would be interesting, for example, to inquire whether the use of material of high. thermal conductivity for the electrodes would be beneficial. But in the magneto the characteristics of the break are fixed. rather by mechanical than electrical considerations, and it is likely that, even if alterations which would prevent arcing rould be suggested, they would be found impracticable. However, it is important to realize that, if arcing can be prevented, the break, which will be sparkless even when there is no primary condenser, is attainable. It is often thought that, unless enormous velocities of separation of the contacts are available, it is quite impossible to break so highly inductive a circuit as the primary of a magneto or induction-coil withont the occurrence of a spark at the break in the absence of a condenser of considerable capacity connected across the terminals. Such an impression is quite erroneous. It is quite easy to construct a break which will carry currents up to 1 amp. without any considerable heating of the electrodes at all; such a break will be sparkless if used for such currents in the primary of any magneto or induction-coil of ordinary construction, even if there is no primary condenser and the speed of separation of the contacts is as low as $20 \mathrm{~cm}$. per sec.

But there is one point which needs further attention. Is the absence of a visible "flash" at the break evidence. that the break is really perfect, and that the interruption of the current occurs instantaneously and without dissipation of energy?

A perfectly definite answer could be given to this question only if it could be shown that the oscillations excited by the break of the primary current agreed with those calculated 
on the assumption that the break was perfect. Unfortunately, however, there are circumstances (connected with the damping of the oscillations) which make an accurate calculation of the oscillations impossible, even on the assumption that the break is perfect. But there seems to be considerable indirect evidence that if the break is "sparkless" it is at least very nearly perfect. For it is found that, so long as no visible flash occurs, the potential developed in the secondary is very nearly proportional to the current broken. in the primary ; but as soon as the limiting current is overstepped and the flash occurs, the potential developed falls at least 30 per cent. The transition from one value of the secondary potential to the other is perfectly sharp; intermediate values are never observed and the lower potential only occurs when a flash is seen. Moreover, so long as no flashing occurs the peak potential obtained when a given current in the primary is broken is perfectly definite and consistent at successive trials; if there is flashing, the values obtained at successive trials are much less regular.

Such observations seem to show that the occurrence of a flash indicates the occurrence of some action which does not occur at all if there is no flash, and that it does not merely indicate an increase of some process which occurs even. when a flash is not seen.

(6) True sparking at the break.-The observations seem also to show that if we suppri'ss areing we are not troubled in normal circumstances with the occurrence of a true spark, as suggested by the theory which we considered originally. But in order to make this conelnsion more certain, it was thought of interest to inquire whether, in any circumstances, true sparking at the break conld be observed.

Such circumstances conld be obtained if, with a current so small that there is no possibility of arcing, the rate of increase of potential can be made greater than the rate of rise of the sparking potential of the gap; accordingly either the rate of rise of potential when a given primary current is broken must be greatly incressed, or the rate of rise of sparking potential greatly reduced. The first is the more convenient alternative to adopt; the necessary conditions are readily secured by increasing greatly the self-inductance of the circuit in which the current is broken. Observations were made, therefore, when the contact to be broken was inserted in the secondary, instead of in the primary, circuit of he magneto.

It was now found that a visible spark occurred at the 
break when the current broken was about 0.02 amp., the speed of the break being about $250 \mathrm{~cm}$./sec. The "spark" now looked like a spark, and did not take the form of the "flash" which appeared when the current was broken in the primary. Further, it could now be definitely established that the limiting current, which could be broken without the appearance of a spark, increased notably with the speed of the break, and, as far as conld be ascertained, it was independent of the form of the terminals and of the pressure between them. All these differences are precisely those which are to be expected if the discharge is now a true spark due to the potential across the gap exceeding the normal spark potential. Howerer, one discrepancy must be noted. 'Though the limiting current increased with the speed of separation, it dic not increase as rapidly as was to be expected. When the speed of separation was doubled, the limiting current increased only some 50 per cent.; the reason for this discrepancy has not been determined.

Observations similar to those shown in fig. 1 were made of the rise of the potential across the gap, both when the spark did occur and when it did not. It was found that, when the spark occurred, the initial part of the curve (corresponding to a time of the order of $10^{-5}$ sec. and a separation of the contacts of the order of $.03 \mathrm{~mm}$.) was precisely the same as when the spark did not occur; but that, at some point on the curve, the readings became wholly irregular and no more consistent measurements could be obtained. The point at which this irregularity sets in is, of course, that at which the spark occurs.

The observations need not be described further. Those already mentioned are sufficient to prove the only fact which is of much importance in this connexion, that the observations when the spark occurs are wholly different from those in the conditions investigated in the earlier experiments and, accordingly, that in those conditions the discharge which is seen to occur is not a true spark. It may be asserted confidently that the conditions necessary for the passage of the true spark are never likely to occur when the primary of a magneto is broken, and that if arcing can be avoided, the break will be perfect.

(7) Note on the liesistance of Contacts.--In the course of these experiments some observations have been made which are of some interest and do not seem to have been described previously.

Suppose that we pass through a contact between two 
metal terminals a gradually increasing current $i$ and measure the potential difference $\mathrm{V}$ between the terminals. Then we shall obtain a relation of the form shown by the full line in fig. 2. In the first stage, $\mathrm{V}$ increases with $i$,

Fig. 2,

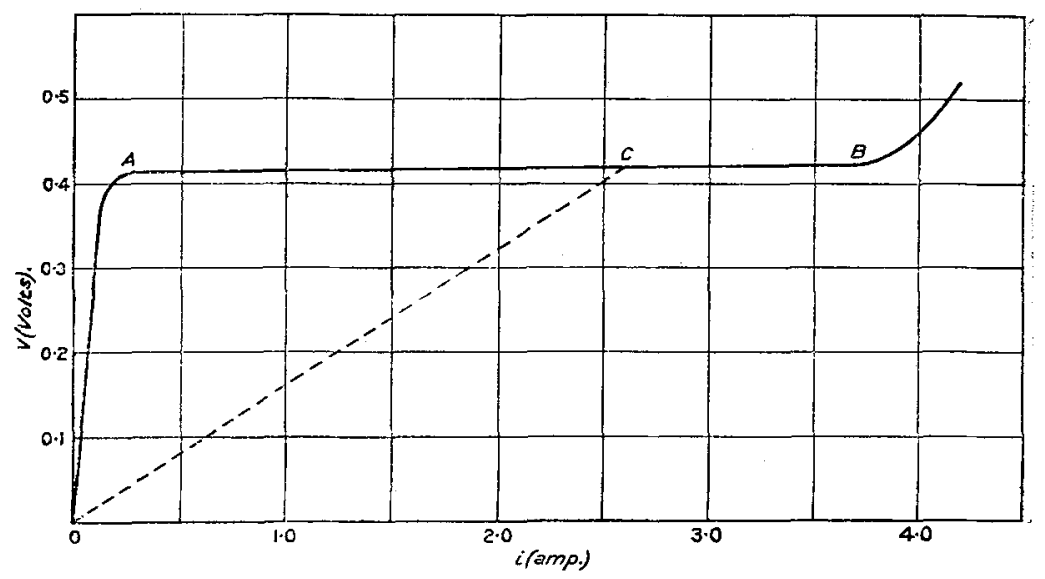

it then becomes independent of $i$ over a considerable range, and later begins to increase once more. The values of $i_{1}$ and $i_{2}$, corresponding to the points $\mathrm{A}$ and $\mathrm{B}$ on the curve, and the constant value of $V\left(V_{0}\right)$ between $A$ and $B$, vary greatly with the nature of the terminals and the pressure between them; but the form of the curve is the same in all. cases investigated.

But now suppose that, having increased $i$ until the point $C$, lying between $A$ and $B$, is reached, we begin to reduce $i$ again, taking great care not to break or shakeviolently the contact; then the values of $V$ obtained will not lie on the Iull line but on the straight dotted iine passing through the origin. I'hat is to say, the contact has now a definite ohmic resistance, $\mathrm{V}_{0} / i_{c}$, determined by $\mathrm{V}_{0}$ and $i_{c}$ tive maximum value of the current which has been passed through the contact. If the contact is broken momentarily the relation between $\mathrm{V}$ and $i$ becomes once more that given by the full line.

No explanation of these observations is offered. It has been recorded before that the resistance of a contact depends. on the current which has been passed through it, and also 
on the time for which the current has passed *, but a definite relation does not seem to have been established. It should be mentioned that as the pressure between the terminals is increased, $V_{0}$ in general decreases and $i_{2}$ increases; it is difficult to determine how $i_{1}$ changes. But no simple relation conld be found between the pressure and the values of $V_{0}$ and $i_{2}$.

An attempt was made to establish a connexion between the form of the full line curve and the least current which would show arcing when the contact was broken. It was thought, for example, that $i_{2}$ might represent this least current. But no simple relation could be found.

\section{Summary.}

1. The notes deal with the question what conditions are necessary to prevent sparking at the break of the primary circuit of an induction-coil or magneto; and, especially, how far the speed of separation of the contucts is important.

2. Experiments show that over a wide range of speeds, the greatest current which can be broken without sparking is independent of the speed of separation of the contacts.

3 . On the other hand, the nature of the contacts separated influences greatly the limiting current.

4. The observations indicate that the "spark" at the break is really an are: this conclusion is confirmed by ineasurements of the potential difference across the contact as it opens. If the discharge is an arc, the independence of the limiting circuit of the speed can be explained.

5. If arcing can be prevented, by preventing heating of the electrodes, the resulting break is perfect.

6. Conditions can be found in which the "spark" at the break is a true spark and not an arc; the limiting current is then dependent of the speed of separation of the contact. But these conditions are not likely to occur in any magneto or induction-coil.

7. Some observations on the resistance of contacts are recorded.

March 1919.

* In the observations of fig. 2 it is supposed that, before taking a measurement of $\mathrm{V}, i$ is kept constant for a time so long that $\mathrm{V}$ reaches a steady value; this time may amount to more than a minute. 